\title{
Overview of Chronic Kidney Disease in Children
}

\author{
Michel Baum \\ Departments of Pediatrics and Internal Medicine, University of Texas Southwestern Medical Center \\ at Dallas, Dallas, Texas 75235-9063
}

There is a growing prevalence of chronic kidney disease world wide. This is in large part due to the fact that in most population's world wide we are getting fatter due to excess caloric intake without a concomitant increase in energy expenditure resulting in an epidemic of obesity and diabetes. In addition our diets are high in sodium and low potassium resulting in a high prevalence of hypertension. According to the United States Renal Data System (USRDS), over 10 percent of people over 20 years of age now have chronic kidney disease. Over 100,000 patients started dialysis last year in the United States making the total of patients on dialysis over 350,000 . The expected mortality of an adult on dialysis is $20 \%$ at one year, $70 \%$ at five years and $90 \%$ by 10 years. The cost for end stage kidney disease is over 30 billion dollars in the United States, impacting not only the patients, but everyone who works and pays for health insurance and pays taxes.

The above statistics are frightening, but reflect renal disease in adults, however we are pediatricians. It is now increasingly apparent that many of the risk factors for chronic kidney disease such as obesity leading to type II diabetes start in childhood and are the harbingers of progressive renal injury as we age. We as pediatricians must be aware of the risk factors and if there are interventions that can be initiated to slow the progression of chronic kidney disease.

The incidence of end stage renal disease in children has increased over the last two decades. According to the USRDS, the number of patients on hemodialysis and peritoneal dialysis has not changed substantively in the last 30 years, but the number of patients who have received renal transplants has increased many fold. In total, the incidence of pediatric patients with end stage renal disease has increased almost 2 fold while the prevalence has increased 4 fold in the last 30 years. While the outlook for pediatric patients with end stage renal disease is not as grim as kidney disease in adults, there is a $20 \% 5$ year mortality in pediatric patients on dialysis. Transplanted pediatric patients fair much better but still have a 5\% mortality at five years.

Chronic kidney disease affects virtually every organ system and in so doing has a major impact on not only on mortality but also on the quality of life of children. The kidney not only clears waste products and maintains fluid and electrolyte homeostasis but is also the site of 25hydroxyvitamin D-1alpha-hydroxylase to generate the active form of vitamin D and erythropoietin for the production of red blood cells. The latter is a major cause for anemia in children with chronic kidney disease that affects exercise tolerance. Children with end stage kidney disease are an immunosuppressed hosts and can expect to be hospitalized twice a year for a total of two weeks, usually the result of an infection. Children with chronic kidney disease tend to grow poorly due to a number of factors. Children with chronic kidney disease often receive erythropoietin and growth hormone injections and take over a half a dozen other medications for blood pressure and electrolyte control. They are on a restricted diet and are

Send Reprint Requests and Correspondence to: Michel Baum, M.D., Department of Pediatrics, U.T. Southwestern Medical Center, 5323 Harry Hines Blvd., Dallas, Texas 75390-9063, PH: (214) 648-3438, FAX: (214) 648-2034, Michel.Baum@UTSouthwestern.edu. 
thus constantly reminded that they are different than other children. Clearly, these factors affect the quality of life of children with kidney disease.

There are many factors that can cause deterioration in renal function such as systemic hypertension and proteinuria. However, the greatest risk factor causing a decline in renal function is renal insufficiency itself. Let me give as an example a laboratory rat. One can take out one kidney on one side and two thirds of the remaining kidney and the rat will appear healthy. There is no inherent renal disease in the remaining kidney. However, over time there is a progressive decline in renal function. Eventually the rat will die of renal failure. The residual glomeruli in that rat will change from a normal appearance to one indistinguishable from focal and segmental glomerulosclerosis. The pathogenesis of the glomerular failure is thought to be due to glomerular capillary hypertension and hypertrophy compensating for the loss of renal mass [1-3]. These overworked glomeruli eventually get worn out and scar resulting in glomerular sclerosis. The same thing happens to human kidneys when there is a significant loss of renal function from any etiology. This clearly does not happen with the loss of one kidney as occurs when we donate a kidney for renal transplantation. However, given the loss of enough renal function, there is inexorable deterioration in renal function over time.

The most common cause for chronic kidney disease in children is obstructive uropathy that is the result of a congenital malformation. Glomerular disease and hypertension are also major causes of chronic kidney disease. What is now apparent is the fact that chronic kidney disease can have its origin due to a prenatal insult [4;5]. Maternal dietary protein deprivation and uteroplacental insufficiency are prevalent worldwide and can lead to infants who are small for gestational age. It has been shown by numerous epidemiologic studies that small for gestational age infants can have a paucity of nephrons and develop hypertension in addition to type II diabetes, dyslipidemia and obesity as adults. These are all harbingers of progressive renal injury. Dr. Vehaskari discusses how prenatal programming causes renal injury and hypertension in his review in this series [6].

Since chronic kidney disease is progressive when there is a significant loss of renal function, it is important to be able to estimate the glomerular filtration rate. To measure glomerular filtration rate, you would want a solute that is completely filtered by the glomerulus and not reabsorbed or secreted by the kidney tubules. Historically, the solute that was used to measure glomerular filtration rate was inulin, but this is a difficult assay to perform and now only used in laboratory animals. There are a number of surrogate ways of estimating renal function such as using the serum creatinine. Creatinine is made in muscle and has a constant level in blood. Creatinine is freely filtered and not reabsorbed, but approximately $10 \%$ of the urine creatinine is secreted by the proximal tubule. Thus, creatinine clearance will overestimate the true glomerular filtration rate. Creatinine has other limitations that especially apply to children. Since creatinine is made in muscle the greater the muscle mass the higher the normal serum creatinine. Thus an adult with a substantive muscle mass may have a normal glomerular filtration rate with a serum creatinine of $1.0 \mathrm{mg} / \mathrm{dl}$ while a child with little muscle mass may have significant renal insufficiency with a creatine of $0.7 \mathrm{mg} / \mathrm{dl}$. In the review in this issue of Current Opinion in Pediatrics, Drs. Staples and Wong discuss new and more accurate ways of estimating glomerular filtration rate (7). This will help clinicians determine who is at risk for progressive kidney injury. In addition, this review will discuss the risk factors for progression of chronic kidney disease.

Since there is no cure for renal injury and renal disease is progressive over time, much work has focused on slowing the progression of renal disease. We have learned a lot about progression of renal disease from the rat with surgical removal 5/6ths of the renal mass. As noted previously, these rats undergo compensatory changes to increase the function of the remaining glomeruli and develop glomerular capillary hypertension. This increase in 
glomerular capillary pressure results in progressive renal injury. Much of our current therapy is aimed at slowing the progression of glomerular injury focuses on ways to lower the glomerular capillary blood pressure. A high protein containing meal is known to increase glomerular capillary pressure by vasodilating the afferent arteriole. Thus, a low protein diet seems like a logical way to prevent progressive renal injury. This works very well in the rat, however clinical studies in humans showed that this was not an effective form of therapy to slow renal disease progression. In addition, it is quite difficult to have patients change their diet. Another approach to decrease glomerular capillary pressure utilized the fact that there are angiotensin II receptors predominantly on the efferent arteriole. Converting enzyme inhibitors or blocking the angiotensin II receptor vasodilate the efferent arteriole and decrease glomerular capillary pressure. This not only slowed the progression of renal insufficiency in rats but in humans as well. Dr. Wuhl provides an outstanding review which includes new information generated in children on how to best slow the progression of renal disease (8).

As chronic kidney disease worsens, conservative medical management eventually becomes inadequate and renal replacement therapy becomes necessary. The indications for dialysis include an electrolyte problem that can't be managed medically (usually hyperkalemia), fluid overload manifesting, not as edema, but as hypertension or congestive hear failure can't be managed medially, or uremia. Uremia presents clinically as the inability to perform ones usual activity, poor appetite and weight loss. Uremia does not correlate well with a specific level of serum creatinine or BUN but in most cases occurs when the renal function deteriorates to approximately 10-15\% of normal renal function. In the review Uri Auron and Patrick Brophy entitled Pediatric Renal Supportive Therapies: The Changing Face of Pediatric Renal Replacement Approaches, they discuss how renal replacement therapy is very much a misnomer (9). Hemodialysis results in the equivalent of about $180 \mathrm{ml} / \mathrm{min}$ of clearance in an adult. While this compares well to the 100-120 $\mathrm{ml} / \mathrm{min}$ in an adult kidney, hemodialysis only occurs 4 hours, 3 times a week giving an average clearance of $10 \mathrm{ml} / \mathrm{min}$. Peritoneal dialysis is performed daily but the clearance is even poorer than hemodialysis. Thus, dialysis is hardly renal replacement therapy. In addition, dialysis does not replace the endocrine functions of the kidney. Dr. Auron and Brophy discuss the limitations of dialysis and how dialysis affects the quality of life of pediatric patients (9).

The only form of real renal replacement therapy is a well functioning renal transplant. A transplant has the potential of replacing $50 \%$ percent of renal function and with compensatory hypertrophy $75 \%$. Transplantation also is not a perfect therapy. One needs to take immunosuppressive therapy to prevent rejection unless one has an identical twin as the donor. These medications increase the risk for opportunistic infections and certain malignancies. In addition calcineurin inhibitors are nephrotoxic. Patients are often at risk for recurrence of the original disease in the transplanted kidney. However, transplantation is currently our best solution and Dr. Sarwal reviews the status of renal transplantation in pediatrics (10).

\section{Reference List}

1. Dunn BR, Anderson S, Brenner BM. The hemodynamic basis of progressive renal disease. Semin Nephrol 1986;6:122-138. [PubMed: 3303239]

2. Anderson S, Brenner BM. The role of intraglomerular pressure in the initiation and progression of renal disease. J Hypertens Suppl 1986;4:S236-S238. [PubMed: 3033176]

3. Anderson S, Meyer TW, Rennke HG, Brenner BM. Control of glomerular hypertension limits glomerular injury in rats with reduced renal mass. J Clin Invest 1985;76:612-619. [PubMed: 2993362]

4. Vehaskari VM, Woods LL. Prenatal programming of hypertension: lessons from experimental models. J Am Soc Nephrol 2005;16:2545-2556. [PubMed: 16049066]

5. Baum M. Role of the Kidney in the Prenatal and Early Postnatal Programming of Hypertension. Am J Physiol Renal Physiol. 2009 
6. Vehaskari. Prenatal programming of kidney disease. Curr Opin Pediatr.

7. Staples, A.; Wong, CS. Risk factors for progression of chronic kidney disease. Curr Opin Pediatr.

8. Wuhl E. Can we slow the progression of chronic kidney disease? Curr Opin Pediatr.

9. Auron A, Brophy PD. Pediatric renal supportive therapies: the changing face of pediatric renal replacement approaches. Curr Opin Pediatr.

10. Sarwal MM. Pediatric Renal Transplantation: An Overview and Update. Curr Opin Pediatr. 